\title{
Applications and New Investigations of the Volta Phase Plate
}

\author{
Kasim Sader $^{1}$,Bart Buijsse ${ }^{1}$, Ilaria Peschiera ${ }^{2}$,Ilaria Ferlenghi ${ }^{2}$ \\ 1. FEI, Achtseweg Noord 5, 5651 GG Eindhoven, The Netherlands \\ 2. Novartis Vaccines, Research Centre, Structural Microscopy, Via Fiorentina 1, 53100 Siena, Italy
}

The use of the Volta Phase Plate for cryoTEM has increased significantly in the last year. Recently two important papers described the novelty of the technique [1] and highlighted the importance of the technique in making new biological discoveries [2]. Here we present our work on both new applications of the Volta Phase Plate to improve imaging of small protein complexes, and new explorations to enhance the information transfer of low coherency sources through the ability to work in focus.

The use of Phase Plates improves the low resolution information content in cryoTEM images, thus enabling the 3D reconstruction of small protein complexes through improved alignment of single particles, similar and synergistic to the improvements obtained by using a counting direct electron detector [3]. The technique has been successfully used to image the NadAV3 (Neisserial adhesion variant 3) homo-trimeric protein and the complex formed by binding to one of its neutralizing antibodies (Figure 1). The NadA protein is one of the components of the recently approved Meningitis B vaccine 4CMenB [4], with the variant 3 being the most important out of all the strains worldwide because it causes the strongest immune response. While the structure of a similar homotrimer from a different variant (NadAV5) has been obtained by X-ray crystallography [4], the structure of NadAV3 has never been determined due to its high flexibility. To increase its low molecular weight (105kDa) the homotrimer has been complexed with Fabs binding to the head region. We used a Titan Krios (300kV) with an FEI Falcon 2/3, Volta Phase Plate, and high dose, and find the contrast to be better than most negative stain images obtained (Figure 1). The structure appears as an elongated rod with one end possibly decorated by $2 / 3$ copies of Fabs. Optimization of the freezing procedure by pre-binding the complex to the carbon, followed by washing and addition of new sample is ongoing.

As an application on the technical side, we have been exploring the use of the Volta Phase Plate to overcome poorly coherent sources (ie. LaB6). Much of the early high resolution unstained biological TEM work [5] was performed with an electron source other than a field emission gun (FEG). This was primarily applied to 2D and helical arrays, as the orientation of the individual subunits of the arrays did not need to be determined relative to one another, and therefore images could be taken very close to focus. This avoided the damping of the CTF due to defocus and the lower spatial coherence of the electron beam. For single particle work, where low resolution information is required for determining orientations of each particle, a substantial defocus $(-2 \mu \mathrm{m})$ is needed to generate the required low resolution frequencies. This normally necessitates a highly coherent electron beam to avoid damping of the CTF, and therefore a FEG. As Phase Plates allow operation in focus, the damping of the CTF is avoided and we are therefore exploring the use of the Volta Phase Plate for single particle analysis (Figure 2). The main challenge is that the on-plane beam covers a large area on the phase plate due to the lower coherency, and it will be more challenging to maintain a constant phase shift close to $\pi / 2$ for the duration of the experiment.

In summary, we describe two important aspects of the use of the Volta Phase Plate: firstly the visualization of small proteins, and in particular antibody-antigen complexes, to aid in the screening of 
possible vaccine candidates. The Volta Phase Plate, together with improvements in direct electron detectors and increased doses, provides a significant improvement in the low resolution signal. Secondly we present our exploration of a method to enable the use of lower coherency sources combined with the Volta Phase Plate for single particle (or tomography) work at medium resolution.

References:

[1] Danev, R. et al. Proceedings of the National Academy of Sciences 111 (2014), 15635-15640.

[2] Asano, S. et al. Science 347 (2015), 439-442.

[3] Lu, P. et al. Nature 512 (2014), 166-170

[4] Malito, E. et al. Proceedings of the National Academy of Sciences 111 (2014), 17128-17133

[5] Henderson, R., Unwin, P.N.T., Nature 257(1975), 28-32.
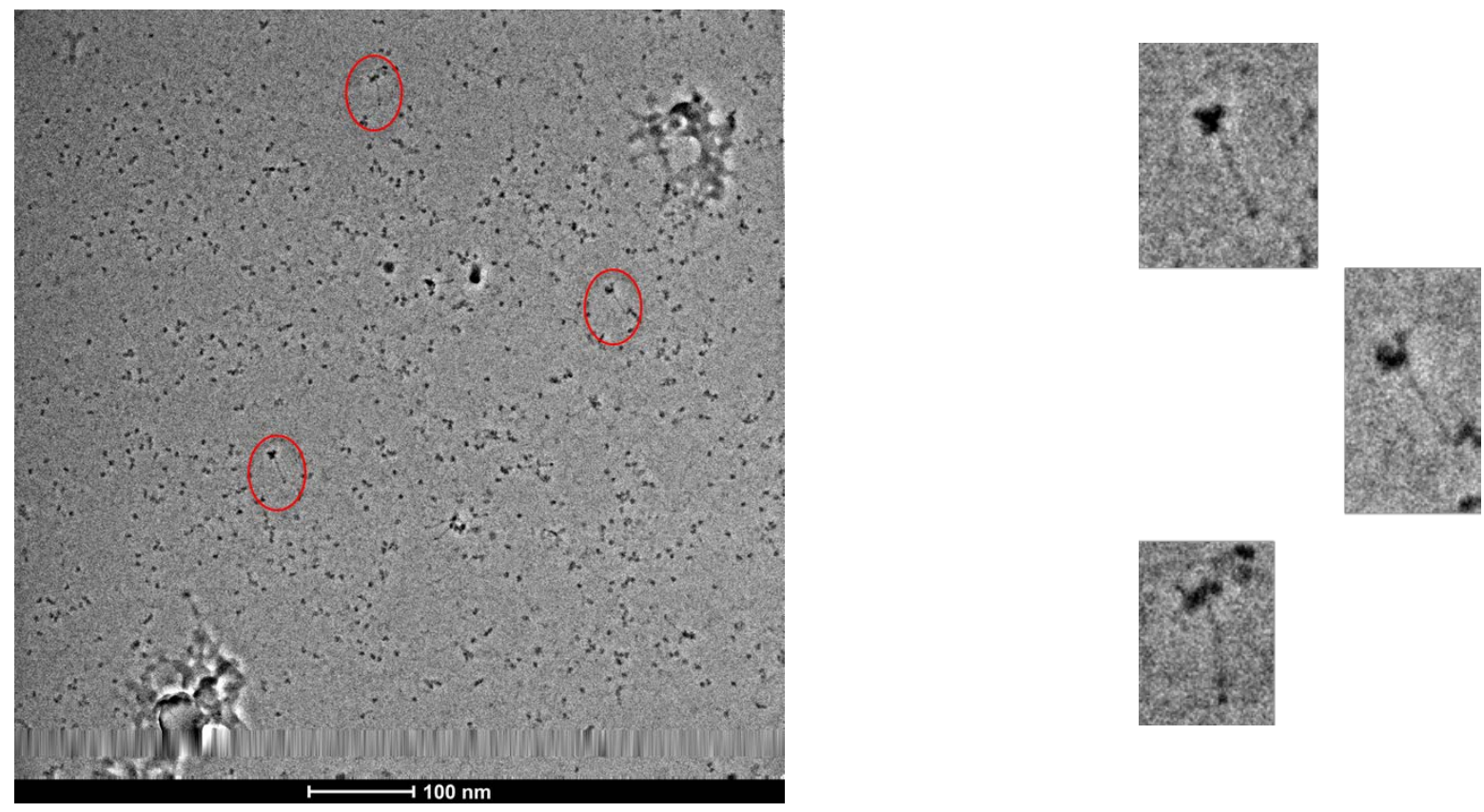

Figure 1. $300 \mathrm{kV}$ Krios, Falcon $2, \sim 100 \mathrm{e}-/ \AA^{2}$, Volta Phase Plate images of the NadAV3-Fab complex with selected molecules magnified.

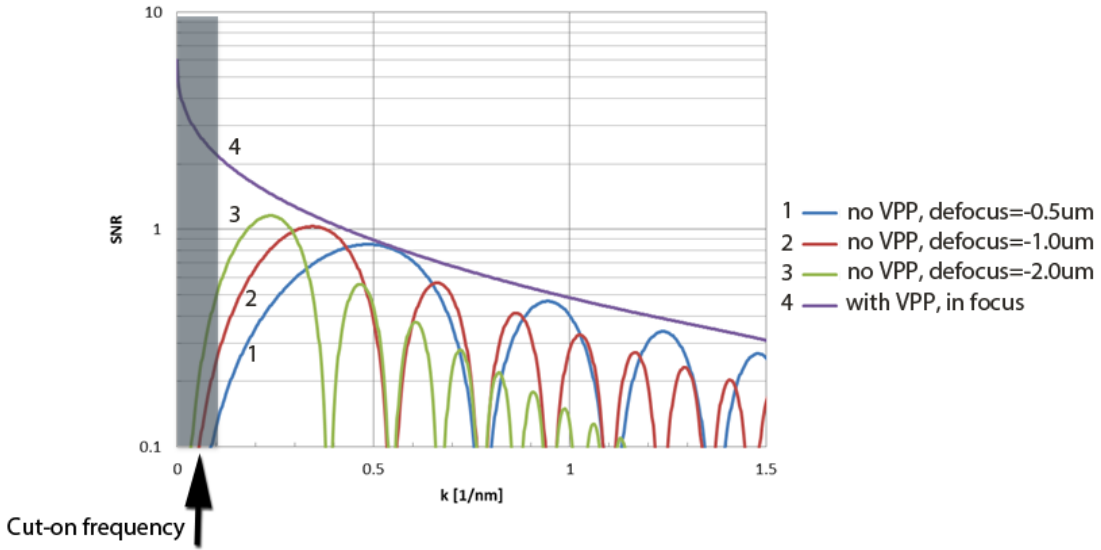

Figure 2. Frequency dependent signal-to-noise ratios derived from experimental data on amorphous carbon images from a LaB6 T20 (30 $\mu \mathrm{m}$ C2), $\sim 10 \mathrm{e} / \AA^{2} / \mathrm{s}$ with and without a Volta Phase Plate (PP). 